July 1995

astro-ph/9507023

Revised February 1996

MPI-PTh/95-63

SFB-375/18

BA- $95-24$

\title{
Nucleon Spin Fluctuations and the Supernova Emission of Neutrinos and Axions*
}

\author{
Hans-Thomas Janka* \\ Department of Astronomy and Astrophysics, The University of Chicago, Chicago, Illinois \\ $60637-1433$ \\ Wolfgang Keil \\ Max-Planck-Institut für Astrophysik, Karl-Schwarzschild-Str. 1, 85740 Garching, Germany \\ Georg Raffelt \\ Max-Planck-Institut für Physik, Föhringer Ring 6, 80805 München, Germany \\ David Seckel \\ Bartol Research Institute, University of Delaware, Newark, Delaware 19716
}

(August 19, 2018)

\begin{abstract}
In the hot and dense medium of a supernova (SN) core, the nucleon spins fluctuate so fast that the axial-vector neutrino opacity and the axion emissivity are expected to be significantly modified. Axions with $m_{a} \lesssim 10^{-2} \mathrm{eV}$ are not excluded by SN 1987A. A substantial transfer of energy in neutrino-nucleon $(\nu N)$ collisions is enabled which may alter the spectra of SN neutrinos relative to calculations where energy-conserving $\nu N$ collisions had been assumed near the neutrinosphere.
\end{abstract}

PACS numbers: 14.60.Lm, 14.80.Mz, 95.30.Cq, 97.60.Bw

Typeset using REVTEX

*To be published in Physical Review Letters 
Introduction. Nucleons interact with each other by a spin-dependent force which causes their spins to flip in a typical nucleon-nucleon $(N N)$ collision. In the hot nuclear medium of a supernova (SN) core where $\rho=10^{14}-10^{15} \mathrm{~g} \mathrm{~cm}^{-3}$ and $T=10-60 \mathrm{MeV}$ the spin-fluctuation rate $\Gamma_{\sigma}$ is estimated to be at least of order $T$. Fluctuating spins act as a source for radiation such as neutrino pairs or axions which couple to the nucleon spin. Also, particles which scatter on nucleons by a spin-dependent force such as neutrinos or other nucleons can gain or lose energy if the target spin fluctuates. Further, fast spin fluctuations cause an averaging of the local spin density which reduces the effective $\nu N$ or $N N$ scattering cross section. To lowest order the physical essence of these processes is described by the Feynman graphs of Fig. 1.1 In a series of papers [1] [3] we have called attention to some of these phenomena and their consequences for the SN emission of neutrinos and axions. In the present note we provide a consistent account of this research and summarize its implications.

The Spin-Density Structure Function. We focus on an isotropic, nonrelativistic, nondegenerate medium of baryon density $n_{B}$, temperature $T$, and a single species of nucleons. The axial-vector neutrino interaction with this medium is governed by the dynamical spin-density structure function (e.g. Refs. [2, [4])

$$
S_{\sigma}(\omega, \mathbf{k})=\frac{4}{3 n_{B}} \int_{-\infty}^{+\infty} d t e^{i \omega t}\langle\boldsymbol{\sigma}(t, \mathbf{k}) \cdot \boldsymbol{\sigma}(0,-\mathbf{k})\rangle
$$

where $\boldsymbol{\sigma}(t, \mathbf{k})$ is the spatial Fourier transform at time $t$ of the nucleon spin-density operator $\boldsymbol{\sigma}(x) \equiv \frac{1}{2} \psi^{\dagger}(x) \boldsymbol{\tau} \psi(x)$. Here $\psi(x)$ is the nucleon field (a Pauli two-spinor) and $\boldsymbol{\tau}$ is a vector of Pauli matrices. The expectation value $\langle\ldots\rangle$ is taken over a thermal ensemble so that detailed balance $S_{\sigma}(\omega, \mathbf{k})=S_{\sigma}(-\omega,-\mathbf{k}) e^{\omega / T}$ is satisfied.

The axial-current transition rate for a neutrino of four momentum $\left(\varepsilon_{1}, \mathbf{k}_{1}\right)$ to $\left(\varepsilon_{2}, \mathbf{k}_{2}\right)$ is then found to be $\frac{1}{4} C_{A}^{2} G_{\mathrm{F}}^{2} n_{B}(3-\cos \theta) S_{\sigma}(\omega, \mathbf{k})$ with $\theta$ the scattering angle, $(\omega, \mathbf{k})=$ $\left(\varepsilon_{1}-\varepsilon_{2}, \mathbf{k}_{1}-\mathbf{k}_{2}\right)$, and $G_{\mathrm{F}}$ the Fermi constant [2]. The axial neutral-current coupling $C_{A}$ to free $p$ 's and $n$ 's should differ somewhat from the charged-current value 1.26 because of the polarization of strange quarks in nucleons. Further, a suppression by $\approx 20 \%$ of all axial couplings is likely in a bulk nuclear medium [2].

We further neglect the momentum transfer from neutrinos to nonrelativistic nucleons (long-wavelength approximation) so that only $S_{\sigma}(\omega)=\lim _{\mathbf{k} \rightarrow 0} S_{\sigma}(\omega, \mathbf{k})$ is used [2]. After an angular integration the axial-current scattering cross section is

$$
\frac{d \sigma_{A}}{d \varepsilon_{2}}=\frac{3 C_{A}^{2} G_{\mathrm{F}}^{2}}{4 \pi} \frac{\varepsilon_{2}^{2} S_{\sigma}\left(\varepsilon_{1}-\varepsilon_{2}\right)}{2 \pi} .
$$

This result is based on the interaction Hamiltonian $\left(C_{A} G_{\mathrm{F}} / 2 \sqrt{2}\right) \bar{\psi}_{N} \gamma_{\mu} \gamma_{5} \psi_{N} \bar{\psi}_{\nu} \gamma^{\mu}\left(1-\gamma_{5}\right) \psi_{\nu}$. Axions a couple to nucleons by $\left(C_{N} / 2 f_{a}\right) \bar{\psi}_{N} \gamma_{\mu} \gamma_{5} \psi_{N} \partial^{\mu} a$ where $C_{N}$ is a model-dependent factor and $f_{a}$ the Peccei-Quinn scale [5]. The axionic energy-loss rate per nucleon is [2]

$$
\frac{Q_{a}}{n_{B}}=\frac{C_{N}^{2}}{(4 \pi)^{2} f_{a}^{2}} \int_{0}^{\infty} d \omega \omega^{4} S_{\sigma}(-\omega) .
$$

\footnotetext{
${ }^{1}$ Fig. [1]b does not provide a complete lowest-order description of "inelastic $\nu N$ scattering" because one needs to include wavefunction and vertex renormalization terms [18]. However, our use of a sum rule obviates the need to include these terms explicitly [19].
} 
Within our approximations, $Q_{a}$ and $\sigma_{A}$ depend on the same function $S_{\sigma}(\omega)$. For a mixture of $n$ and $p$ the nuclear response is characterized by a linear combination of a small number of functions to allow for the isospin dependence of different processes.

In a noninteracting medium the nucleon spins do not evolve, and so in Eq. (1) $\boldsymbol{\sigma}(t)=$ $\boldsymbol{\sigma}(0)$. Then the time integration yields $S_{\sigma}(\omega)=2 \pi \delta(\omega)$. Indeed, if one ignores recoil effects the neutrino cannot gain or lose energy in a $\nu N$ collision. Also, $Q_{a}=0$ in agreement with the fact that free nucleons do not emit radiation.

Nucleon-nucleon collisions with a spin-dependent force cause a nontrivial evolution $\boldsymbol{\sigma}(t)$. Still, in $\int_{-\infty}^{+\infty} d \omega S_{\sigma}(\omega)$ the $e^{i \omega t}$ factor gives us $2 \pi \delta(t)$. Therefore, one finds that the "sum" $\int_{-\infty}^{+\infty} d \omega S_{\sigma}(\omega)=\left(4 / 3 n_{B}\right) 2 \pi\langle\boldsymbol{\sigma}(0) \cdot \boldsymbol{\sigma}(0)\rangle$ is independent of the time evolution of $\boldsymbol{\sigma}(t)$. Therefore, unless the $N N$ interaction establishes spin-spin correlations which are absent in a noninteracting medium, one finds the sum rule $\int_{-\infty}^{+\infty} d \omega S_{\sigma}(\omega)=2 \pi$.

In Ref. [6] the static spin-density structure function $S_{\sigma}(\mathbf{k})=\int_{-\infty}^{+\infty} S_{\sigma}(\omega, \mathbf{k}) d \omega / 2 \pi$, and notably its long-wavelength limit $\mathbf{k} \rightarrow 0$, has been used to study correlation effects for SN neutrino opacities. We believe that it is crucial to keep the dynamical structure because the $\omega$-dependence is not essentially $\delta(\omega)$. Still, the results of [6] indicate a significant reduction of $S_{\sigma}(\mathbf{k})$ due to $N N$ interactions so that the partial pairing of spins may reduce $\int_{-\infty}^{+\infty} d \omega S_{\sigma}(\omega)$ below its free value $2 \pi$.

Ansatz for $S_{\sigma}(\omega)$. Motivated by a classical bremsstrahlung calculation we assume a functional form $S_{\sigma}(\omega)=\Gamma_{\sigma} \omega^{-2} s(\omega / T) b(\omega / T)$ where $\Gamma_{\sigma}$ is the spin-fluctuation rate, $s(x)$ a dimensionless even function normalized such that $s(0)=1$, and $b(x)=1$ for $x>0$ and $e^{x}$ for $x<0$ to satisfy detailed balance. If one models the $N N$ interaction by a one-pion exchange (OPE) potential one finds for a single species of nucleons [2,7]

$$
\Gamma_{\sigma, \mathrm{OPE}}=4 \sqrt{\pi} \alpha_{\pi}^{2} \frac{n_{B} T^{1 / 2}}{m_{N}^{5 / 2}}=8.6 \mathrm{MeV} \rho_{13} T_{10}^{1 / 2},
$$

where $\alpha_{\pi} \equiv\left(f 2 m_{N} / m_{\pi}\right)^{2} / 4 \pi \approx 15$ with $f \approx 1.0$ is the pion fine structure constant, $\rho_{13} \equiv$ $\rho / 10^{13} \mathrm{~g} \mathrm{~cm}^{-3}, T_{10} \equiv T / 10 \mathrm{MeV}$, and the vacuum value $m_{N}=940 \mathrm{MeV}$ has been used. In the following we shall use the classical result $s(x)=1$. The detailed large- $x$ behavior depends on the assumed short-range behavior of the $N N$ interaction potential, but the OPE potential is too singular at short distances to yield a useful answer.

For $\omega \lesssim \Gamma_{\sigma}$ the behavior of $S_{\sigma}(\omega)$ is dominated by multiple-scattering effects. A proper calculation in this regime does not exist, but two of us (RS) have previously argued that a plausible representation is [1, 2]

$$
S_{\sigma}(\omega) \rightarrow \frac{\Gamma_{\sigma}}{\omega^{2}+\Gamma^{2} / 4} s(\omega / T),
$$

with $\Gamma \approx \Gamma_{\sigma}$. Taking $s(x)=1$ the sum rule is satisfied if one chooses $\Gamma=\Gamma_{\sigma}$ for $\Gamma_{\sigma} \ll T$, and $\Gamma=\Gamma_{\sigma} / 2$ for $\Gamma_{\sigma} \gg T$. Assuming this form for $S_{\sigma}(\omega)$ allows us to compute the variation of the thermally averaged axial-current scattering cross section $\left\langle\sigma_{A}\right\rangle$, and of $Q_{a} / n_{B}$, with $\Gamma_{\sigma}$ (Fig. 2). In a dilute medium $\left(\Gamma_{\sigma} \ll T\right),\left\langle\sigma_{A}\right\rangle$ is independent of density. The axion emission rate per nucleon $Q_{a} / n_{B}$ is proportional to $n_{B}$ and thus to $\Gamma_{\sigma}$. Such conditions may be relevant near the "neutrinosphere" where $\rho_{13} \approx 0.1$. However, for the conditions in a SN core Eq. (4) yields $\Gamma_{\sigma, \mathrm{OPE}} / T=20-50$ [3]. While we will argue that this perturbative result 
likely is an overestimate of the true $\Gamma_{\sigma}$ it still indicates that multiple-scattering effects may be expected to suppress both $\left\langle\sigma_{A}\right\rangle$ and $Q_{a} / n_{B}$.

"Calibration" of $\Gamma_{\sigma}$ at High Density. In the ansatz Eq. (5) for $S_{\sigma}$ it was implicitly assumed that $\Gamma_{\sigma}$ increases linearly with density, as for $\Gamma_{\sigma, \mathrm{OPE}}$. It is unlikely that this is correct - a similar "averaging effect" which reduces the $\nu N$ cross section also affects $N N$ collisions. For $\Gamma_{\sigma, \mathrm{OPE}} \gtrsim T$, one should consider the true $\Gamma_{\sigma}$ to be a slowly increasing, but unknown, function of the density.

The neutrino signal of SN 1987A offers a unique possibility to test for the actual behavior of $\Gamma_{\sigma}$. The neutrino signal of a SN depends sensitively on the neutrino opacity at high density, which is dominated by $\sigma_{A}$. Since $\sigma_{A}$ drops precipitously if $\Gamma_{\sigma} \gtrsim T$, we in principle have an experimental probe of $\Gamma_{\sigma}(\rho, T)$. To this end, three of us (KJR) have performed detailed numerical calculations of the cooling of newborn neutron stars with modified axial-vector neutrino opacities [3]. The result of this study is that reduced neutrino opacities shorten the neutrino pulse dramatically and increase the number of events and the event energies in the Kamiokande II and IMB detectors. The best fit to the data is achieved if one ignores opacity suppression effects entirely. An overall reduction by more than $50 \%$ seems to be excluded. This is still compatible with the expected reduction of $C_{A}$ in a bulk nuclear medium which entails an opacity suppression by 30-40\% [2], but would be incompatible with any significant reduction due to either "pairing" of nucleon spins by the $N N$ interaction or broadening of $S_{\sigma}(\omega)$. Similar conclusions follow from Ref. [8] where the opacity was indirectly lowered by increasing the number of neutrino species.

Of course, the SN 1987A observations encompass very few events. Probably the most reliable measure of the opacities in the deep interior is the neutrino pulse duration as the number of events and their energies are largely determined by the spectral features of the neutrino flux and thus by the conditions near the neutron star surface. It is worrying that a long signal and thus large opacities are mostly supported by the three late Kamiokande events which are separated from the first eight by a $7 \mathrm{~s}$ break. If the late events had some other cause besides the Kelvin-Helmholtz cooling such as late-time accretion, possibly triggered by a reverse-shock [9], then reduced opacities might become more tolerable.

Barring this possibility we infer that $\Gamma_{\sigma}$ cannot grow linearly with density but reaches a value of at most a few times $T$ in a SN core. Values for $\Gamma_{\sigma}$ to the right of the hatched band in Fig. 2 seem to be excluded. If correct, this result implies that the OPE value for $\Gamma_{\sigma}$ is an overestimate.

Axion Bounds. Previous axion bounds from SN 1987A had been based on the "naive emission rate" (dashed line in Fig. 2), and had employed a perturbative estimate of $\Gamma_{\sigma}$. During the first few seconds after collapse $\Gamma_{\sigma, \mathrm{OPE}} / T=20-50$ throughout the SN core. This would take one somewhat to the right of the maximum of the solid line in Fig. 2. If our "calibration" of $\Gamma_{\sigma}$ is correct, one is somewhat to the left of the maximum. Either way, $Q_{a}$ is near its theoretical maximum so that the "naive" $Q_{a}$ is suppressed by about a factor of 10. Because $Q_{a}$ is proportional to the square of the axion mass, previous limits on $m_{a}$ are relaxed by about a factor of 3 .

The often-quoted limit $m_{a} \lesssim 10^{-3} \mathrm{eV}$ was based on $C_{N}=0.5$ for $N=p$ and $n$ [10. In popular axion models these couplings are much smaller, for example $C_{n} \approx 0$ in the socalled KSVZ model. Therefore, in a detailed review [5] it was shown that translating the SN 1987A bound on the $a N$ coupling into a bound on $m_{a}$ really yields $m_{a} \lesssim 3 \times 10^{-3} \mathrm{eV}$ in 
the KSVZ and other typical models. Together with the above suppression effect we conclude that $m_{a} \lesssim 10^{-2} \mathrm{eV}$ is not excluded.

Energy Transfer in $\nu N$ Collisions. In previous SN studies of neutrino transport, $\nu N$ collisions have been taken to be energy conserving [11, 12] because $S(\omega) \propto \delta(\omega)$ for scattering of $\nu$ 's from single, "heavy" nucleons. The $\nu_{\mu}$ (and $\bar{\nu}_{\mu}, \nu_{\tau}$, and $\bar{\nu}_{\tau}$ ) spectra are formed at the radius where $\nu e$ scattering and $\nu_{\mu} \bar{\nu}_{\mu} \leftrightarrow e^{-} e^{+}$become inefficient for transferring energy between the neutrinos and the medium. However, the $\nu_{\mu}$ 's must diffuse through a considerable overburden of nuclear material before they escape. In general this material will have a temperature $T$ that is lower than the temperature $T_{\nu}$ that characterizes the $\nu_{\mu}$ spectrum. Thus the average $\nu_{\mu}$ energies are usually found to be a factor of 1.3-1.8 larger than those of $\bar{\nu}_{e}$ 's, which react via $\beta$-processes and decouple at lower density and temperature.

A broad $S_{\sigma}(\omega)$ such as in Eq. (5) would allow for energy transfer between the neutrinos and the medium. Further, even for $\nu N$ processes the $\delta$ function is only an approximation that neglects recoil corrections. Their inclusion would modify the expected $\nu_{\mu}, \bar{\nu}_{\mu}, \nu_{\tau}$, and $\bar{\nu}_{\tau}$ spectra. In turn, energy conservation and a reduced energy flux in these flavors would imply increased $\nu_{e}$ and $\bar{\nu}_{e}$ energy and number fluxes. This is important in planning for detection of SN neutrinos at Superkamiokande or SNO, for understanding the explosion mechanism of Type II supernovae [14, 15], and for the conjecture that r-process nucleosynthesis occurs in the high-entropy medium that surrounds the protoneutron star [16].

For neutrinos with a black-body spectrum the average recoil energy transfer in $\nu N$ collisions with nondegenerate nucleons is $\langle\Delta \varepsilon\rangle_{\nu N} \approx 30 T_{\nu}\left(T-T_{\nu}\right) / m_{N}$ [12, 13], and the average energy exchange $\langle\Delta \varepsilon\rangle_{\nu N N}$ in inelastic $\nu N N$ scatterings is

$$
\frac{\int_{0}^{\infty} d \varepsilon_{1} \varepsilon_{1}^{2} e^{-\varepsilon_{1} / T_{\nu}} \int_{0}^{\infty} d \varepsilon_{2} \varepsilon_{2}^{2}\left(\varepsilon_{2}-\varepsilon_{1}\right) S_{\sigma}\left(\varepsilon_{1}-\varepsilon_{2}\right)}{\int_{0}^{\infty} d \varepsilon_{1} \varepsilon_{1}^{2} e^{-\varepsilon_{1} / T_{\nu}} \int_{0}^{\infty} d \varepsilon_{2} \varepsilon_{2}^{2} S_{\sigma}\left(\varepsilon_{1}-\varepsilon_{2}\right)} .
$$

With Eq. (5), the sum rule for $S_{\sigma}(\omega)$, and $s(x)=1$ we find numerically $\langle\Delta \varepsilon\rangle_{\nu N N}=\zeta \frac{1}{4} \Gamma_{\sigma}(T-$ $\left.T_{\nu}\right)\left(T_{\nu} T\right)^{-1 / 2}$ where $\zeta^{-1} \approx 1+\log _{10}\left(1+\Gamma_{\sigma} / T\right)$ within $15 \%$ for $1<T_{\nu} / T<2$ and $0.1<$ $\Gamma_{\sigma} / T<10$. For conditions near the neutrinosphere both the $\nu N$ and $\nu N N$ processes can therefore transfer several $\mathrm{MeV}$ of energy to the medium. Even though the energy exchange per interaction may be small, a typical neutrino experiences 10-100 interactions during the diffusion process through the protoneutron star surface layers. The net effect may lead to substantially lower average energies of the $\nu_{\mu}$ 's and $\bar{\nu}_{\mu}$ 's. Thus, both $\nu N$ recoil and the inelastic $\nu N N$ modes of energy transfer are potentially important near the neutrinosphere where the spectra are formed.

The same conclusion is reached by comparing the recoil energy transfer rates between neutrinos and a medium of nucleons and between neutrinos and a medium of degenerate electrons [12,

$$
\frac{Q_{\nu N}}{Q_{\nu e}} \approx 0.8 \frac{1+5 C_{A}^{2}}{2 C_{e}} \rho_{13}^{-1 / 3}\left(\frac{T_{\nu}}{10 \mathrm{MeV}}\right)^{2}\left(\frac{Y_{e}}{0.1}\right)^{-4 / 3} .
$$

Here, the effective weak interaction constant $C_{e}$ for $\nu$ scattering is $0.33\left(\nu_{\mu, \tau}\right)$, and 0.26 $\left(\bar{\nu}_{\mu, \tau}\right)$. In the neutron star surface layers $\rho \approx 10^{12}-10^{13} \mathrm{~g} / \mathrm{cm}^{3}$, and $Y_{e}$ drops to $0.05-0.1$ within about a second after stellar core collapse [17]. The energy transfer from $\nu_{\mu}$ to the medium is apparently dominated by direct transfer to the nucleons by an order of magnitude 
or greater even though the calculation behind Eq. (7) does not include the full effects of electron phase-space blocking.

Summary. For axial-vector current interactions of neutrinos and axions in a SN core multiple-scattering effects must not be ignored. Notably, the spin-fluctuation rate cannot be simultaneously very small $\left(\Gamma_{\sigma} \ll T\right)$, as had been assumed for the neutrino opacities, and very large $\left(\Gamma_{\sigma} \gg T\right)$, as had been assumed for the axion emissivities. Our study suggests that it is in the middle, i.e. of order $T$, a range where the impact on neutrino opacities is relatively mild while the axion emissivity is near its maximum allowed by multiple-scattering effects (but still less than previous calculations had indicated). At the present state of the art, the neutrino opacities cannot be calculated from first principles within controlled approximations. Therefore, the SN neutrino signal duration and other signal characteristics can be predicted only with the accuracy of a dimensional analysis, implying uncertainties for models of the $\nu$-driven mechanism of Type II SN explosions [14. Even near the neutrinosphere nonnegligible effects such as the nonconservation of energy in $\nu N$ collisions are important where both the recoil and the bremsstrahlung mode of $\nu N$ energy transfer need to be included. A quantitative understanding of the impact of energy transfer in $\nu N$ collisions on the expected neutrino fluxes and spectra will require detailed numerical simulations.

Note Added. After this paper had been submitted, a perturbative calculation of the reduction of weak scattering rates by nucleon (iso)spin fluctuation has appeared [18]. In essence, this is a calculation of the slope of our $\left\langle\sigma_{A}\right\rangle$ curve in Fig. 2 at the point $\Gamma_{\sigma} / T=0$. In linear-response theory, this slope is independent of our Lorentzian ansatz and agrees with the perturbative result - for a detailed comparison see Ref. [19]. Further, Sigl [20] has derived an f-sum rule for $S_{\sigma}(\omega)$ which allows one to estimate an upper limit on $\Gamma_{\sigma}$, providing a theoretical underpinning for our SN 1987A bound on $\Gamma_{\sigma} / T$.

Acknowledgements. At the University of Chicago this research was supported by NSF grant AST 92-17969, NASA grant NAG 5-2081, and by an Otto Hahn Postdoctoral Scholarship of the Max-Planck-Society. At the Max-Planck-Institutes, partial support by the European Union contract CHRX-CT93-0120 and by the Deutsche Forschungsgemeinschaft grant SFB 375 is acknowledged, and at Bartol by DOE grant DE-AC02-78ER05007. 


\section{REFERENCES}

* On leave from the MPI für Astrophysik, Garching.

[1] G. Raffelt and D. Seckel, Phys. Rev. Lett. 67, 2605 (1991).

[2] G. Raffelt and D. Seckel, Phys. Rev. D 52, 1780 (1995).

[3] W. Keil, H.-Th. Janka, and G. Raffelt, Phys. Rev. D 51, 6635 (1995).

[4] N. Iwamoto and C. J. Pethick, Phys. Rev. D 25, 313 (1979).

[5] For a review see G. G. Raffelt, Phys. Rep. 198, 1 (1990).

[6] R.F. Sawyer, Phys. Rev. C 40, 865 (1989).

[7] R.P. Brinkmann and M.S. Turner, Phys. Rev. D 38, 2338 (1988).

[8] A. Burrows, M.T. Ressell, and M.S. Turner, Phys. Rev. D 42, 3297 (1990).

[9] H.-Th. Janka, Report astro-ph/9505034, talk presented at the SFB-meeting on AstroParticle-Physics, Ringberg Castle, Tegernsee, Germany, March 6-10, 1995.

[10] A. Burrows, M.S. Turner, and R.P. Brinkmann, Phys. Rev. D 39, 1020 (1989) and references therein.

[11] R.W. Mayle, J.R. Wilson, and D.N. Schramm, Astrophys. J. 318, 288 (1987). S.W. Bruenn, Phys. Rev. Lett. 59, 938 (1987). H.-Th. Janka and W. Hillebrandt, Astron. Astrophys. Suppl. 78, 375 (1989) and Astron. Astrophys. 224, 49 (1989). E.S. Myra and A. Burrows, Astrophys. J. 364, 222 (1990).

[12] H.-Th. Janka, Ph.D. Thesis, Technische Univ. München (1991, Report MPA-587) and references therein.

[13] D.L. Tubbs, Astrophys. J. 231, 846 (1979).

[14] H.A. Bethe and J.R. Wilson, Astrophys. J. 295, 14 (1985). H.-Th. Janka, in Frontier Objects in Astrophysics and Particle Physics, edited by F. Giovannelli and G. Mannocchi, (Società Italiana di Fisica, Bologna, 1993), p. 345 and references therein.

[15] G.M. Fuller et al., Astrophys. J. 389, 517 (1992).

[16] K. Takahashi, J. Witti, and H.-Th. Janka, Astron. Astrophys. 286, 857 (1994). S.E. Woosley et al., Astrophys. J. 433, 229 (1994). B.S. Meyer, preprint, to appear in Astrophys. J. Letters.

[17] A. Burrows and J.M. Lattimer, Astrophys. J. 307, 178 (1986). J.R. Wilson and R.W. Mayle, in The Nuclear Equation of State, Part A, edited by W. Greiner and H. Stöcker (Plenum Press, New York, 1989), p. 731. W. Keil and H.-Th. Janka, Astron. Astrophys. 296, 145 (1995).

[18] R.F. Sawyer, Phys. Rev. Lett. 75, 2260 (1995).

[19] G. Raffelt, G. Sigl, and D. Seckel, Report MPI-PTh/95-130, submitted to Phys. Rev. D.

[20] G. Sigl, Report Fermilab-Pub-95/274-A, to be published in Phys. Rev. Lett. (1996). 


\section{FIGURES}

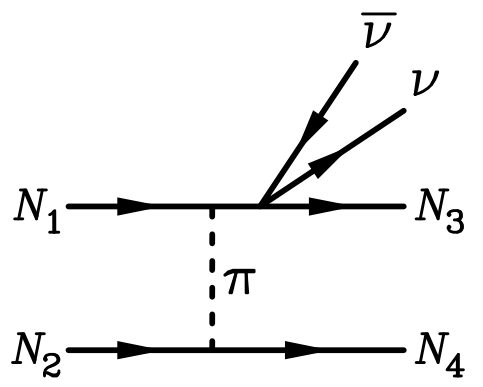

(a)

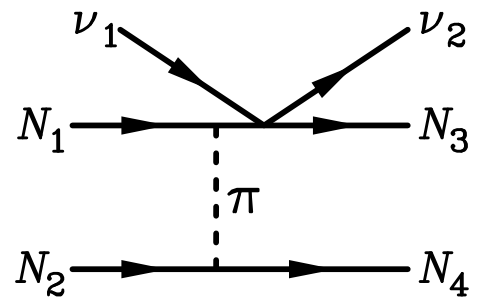

(b)

FIG. 1. (a) Bremsstrahlung emission of neutrino pairs in $N N$ collisions. Instead of a neutrino pair, an axion can be emitted. (b) "Inelastic $\nu N$ scattering" in the presence of a bystander nucleon.

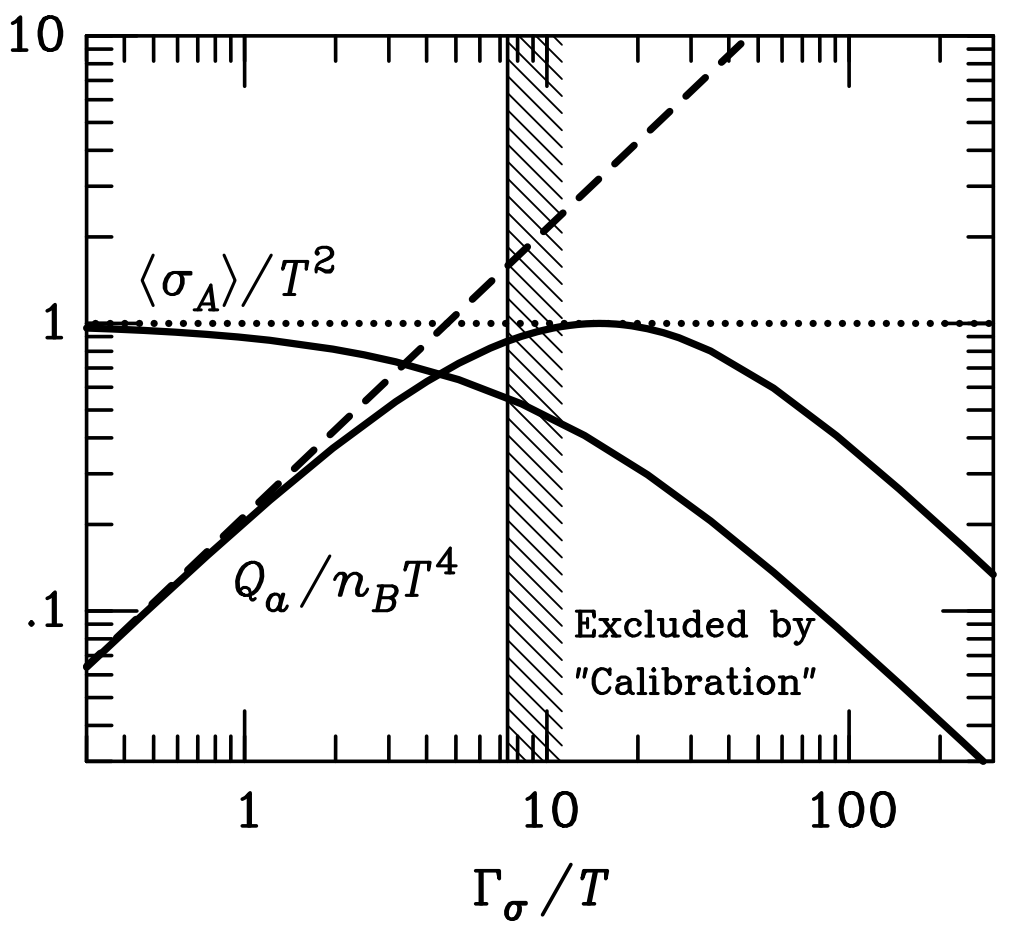

FIG. 2. Schematic dependence of the average axial-vector $\nu N$ scattering cross section $\left\langle\sigma_{A}\right\rangle$ and of the axion emission rate per nucleon $Q_{a} / n_{B}$ on $\Gamma_{\sigma}$. The broken lines correspond to the naive results which are characterized by $\Gamma=0$ in Eq. (5) for $Q_{a} / n_{B}$ and by $S_{\sigma}(\omega)=2 \pi \delta(\omega)$ for $\left\langle\sigma_{A}\right\rangle$. 